EPJ Web of Conferences 110,01083 (2016)

DOI: $10.1051 /$ epjconf/201611001083

(C) Owned by the authors, published by EDP Sciences, 2016

\title{
INVESTIGATION OF THE EXTINGUISHING FEATURES FOR LIQUID FUELS AND ORGANIC FLAMMABLE LIQUIDS ATOMIZED BY A WATER FLOW
}

\author{
Ivan V. Voytkov ${ }^{1}$, Maksim V. Zabelin ${ }^{1}$, Olga V. Vysokomornaya ${ }^{1, \text { a }}$ \\ ${ }^{1}$ National Research Tomsk Polytechnic University, 634050 Tomsk, Russia
}

\begin{abstract}
The processes of heat and mass transfer were investigated experimentally while moving and evaporating the atomized water flow in high-temperature combustion products of typical liquid fuels and organic flammable liquids: gasoline, kerosene, acetone, crude oil, industrial alcohol. We determined typical periods of liquid extinguishing by an atomized water flow of various dispersability. Data of the discharge of extinguishing medium corresponding to various parameters of atomization and duration of using the atomization devices was presented. It is shown that $U_{\mathrm{m}} \approx 3.5 \mathrm{~m} / \mathrm{s}$ is a minimal outflow velocity of droplets during moving while passing the distance of $1 \mathrm{~m}$ in the hightemperature gas medium to stop the combustion of organic liquids.
\end{abstract}

\section{Introduction}

Technological facilities and industrial estates of oil and gas extraction and petrochemical industries are characterized by an increased risk of unregulated fires. Usually, fires at such estates fall into increased complexity due to the dissemination of an ignition source over large areas, low flammable level and extreme explosion risk of organic liquids and fuels, significant energy release from the combustion of oil and products of its processing [1,2].

When extinguishing fires of oil and petroleum products by atomized liquid flows (water, solutions, emulsions and suspensions based on it), the rate of evaporation of extinguishing composition in the high-temperature combustion products is a determinative one. The water vapors resulting from phase transformation, on the one hand, reduce the temperature of ignition source due to the expenditure of energy for evaporation, and, on the other hand, displace the oxidant from the fire zone.

The results of experiments [3] suggest the possibility of using the liquid extinguishing compositions based on water to suppress the combustion of liquid fuels and organic combustible liquids, considering restrictions for certain parameters of atomizing the extinguishing medium (stepwise atomization of droplets of varying dispersion) in order to avoid overspending of water to extinguish by "large" droplets and entrainment of extinguishing medium particles with a typical size less than $10 \mu \mathrm{m}$ by opposite high-temperature gas flow of quenching medium particles with a typical size less than 10 microns [3].

It is of interest to estimate the consumption, as well as typical times of atomizing the extinguishing liquid to the moment of liquidation of fire in the forming a flow of different dispersion.

a Corresponding author: vysokomornaja@tpu.ru

This is an Open Access article distributed under the terms of the Creative Commons Attribution License 4.0, which permits unrestricted use, distribution, and reproduction in any medium, provided the original work is properly cited. 
The purpose of the work is to investigate experimentally establishing the extinguishing periods of fires of typical flammable liquids by the polydisperse droplet flow of water with controlled parameters of atomization (sizes of droplets in a flow $\left(R_{\mathrm{d}}\right)$, velocity of droplet flow $\left(U_{\mathrm{m}}\right)$, component composition of extinguishing liquid).

\section{Experimental setup and procedure}

In conducting the experimental investigations, we used the setup which is similar to that presented in the works $[4,5]$. The main equipment being a part of the experimental setup includes generator of laser emission, two-pulse Nd:YAG laser (wavelength $-532 \cdot 10^{-9} \mathrm{~m}$, minimal energy in a pulse $70 \cdot 10^{-3} \mathrm{~J}$, maximal duration of a pulse $-12 \cdot 10^{-9} \mathrm{~s}$, repetition frequency $-15 \mathrm{~Hz}$ ), sync processor, cross-correlation (picture format - 2048x2048 pixels, frame frequency $-1.5 \mathrm{~Hz}$, minimal delay between frames $-5 \cdot 10^{-6} \mathrm{~s}$ ) and high-speed video cameras (shooting speed is not less than $10^{5}$ frames per second (when the definition is $640 \times 480$ pixels)). In experimentations we applied several optical methods of droplet flow diagnostic (PIV, PTP, Stereo PIV, SP) together with corresponding hardware and software for processing the results of experiments.

The atomizer, that turns according to necessary atomization parameters (in the experiments we used special metal pulverizers), was connected through the channel with the outlet of vessel containing water with known parameters and characteristics. Depending on the type of experiment, we chose the height for installation of cross-correlation video cameras and laser (in the case of measuring by PIV and PTV the installation height of video camera and laser were chosen so that the optical axis of the camera and the plane of the light sheet of laser intersect at an angle of 90 degrees in the registration area; in the case of measuring by Stereo PIV method - the angle between the optical axes of the cameras and the plane of the light sheet of laser is 30-60 degrees; in the case of using SP method in front the camera we set the strobe light - special diffuse screen, connected by a fiber with a laser, a mounting height of laser in this case is not important). The base of a hollow cylinder was filled with typical liquid petroleum product (about $250 \mathrm{ml}$ ), which was initiated by the ignition before the experimentation. When the temperature of the combustion products $T_{\mathrm{g}}$ reaches the value of about $1100 \mathrm{~K}$, the atomizer fixed at a distance of $0.5 \mathrm{~m}$ from the upper face of the cylinder was switched. The specialized software (realizing the optical methods as PIV [6], PTV [7], Stereo PIV [8] or SP [9]) started on the PC, and the motion of the atomized flow of suspension was recorded by video (it was at the inlet and outlet from a cylindrical channel). Characteristic extinguishing time of typical flammable substances $t_{\mathrm{e}}$ was the main parameter determined in experimentations. Extinguishing was considered as achieved while reducing the temperature of the gaseous medium, measured by thermocouples installed in the region above the surface of liquid fuel, by $50 \%$ or more within two minutes.

Gasoline ON-92, aviation kerosene TS-1, acetone (dimethyl ketone), crude oil (from the Chayandinskoye field with a density of $\rho \approx 667 \mathrm{~kg} / \mathrm{m}^{3}$, industrial alcohol (methanol) were used as typical liquid fuels.

\section{Results and Discussion}

For atomization pulverizers applied in experiments, we defined the characteristics of atomization: size of the generated droplets $R_{\mathrm{m}}$ and discharge intensity of liquid $\mu_{\mathrm{w}}$ by pulverizer per unit time. Fig. 1 illustrates the dependence of liquid volume expended by pulverizer of each type on the length of atomization time and initial sizes of droplets. 


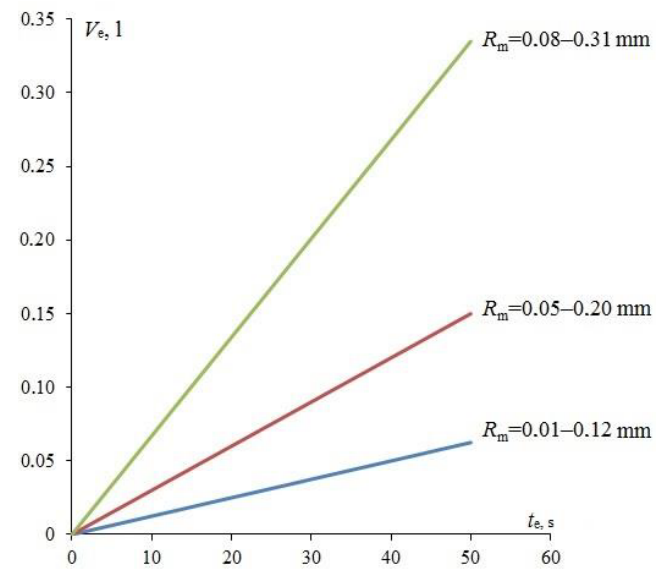

$a$

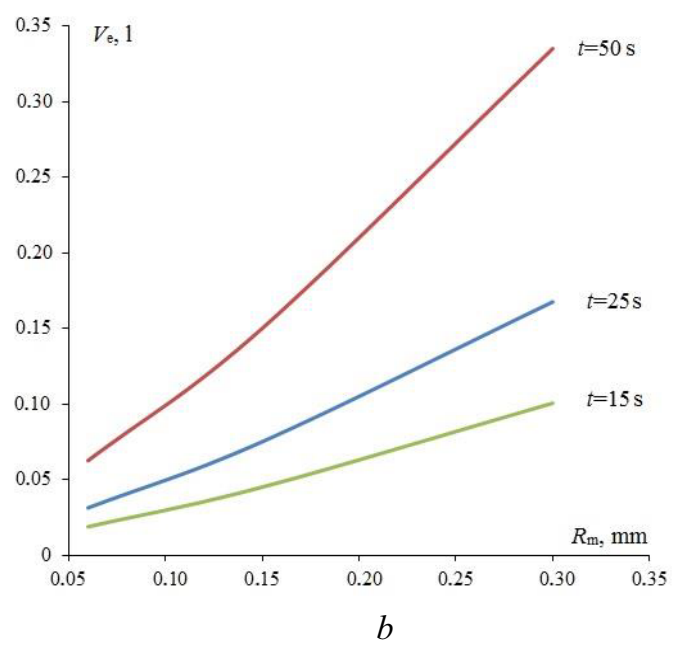

Figure 1. Approximation dependences of liquid volume $V_{\mathrm{e}}$ expended by pulverizers on the characteristic times of atomization $t_{\mathrm{e}}$ for different values of $R_{\mathrm{m}}(a)$ and initial sizes of droplets $R_{\mathrm{m}}$ for various lengths of atomization times (b) at $U_{\mathrm{m}} \approx 2 \mathrm{~m} / \mathrm{s}$.

The characteristic curves presented in Fig. 1 set up a correspondence between volume flow of extinguishing composition, characteristic size of droplets of atomized liquid and using a length of atomizer.

Experimental investigations of heat and mass transfer while moving the atomized water flow in high-temperature combustion products of flammable liquids allowed defining the typical extinguishing times $t_{\mathrm{e}}$ for typical combustible substances (gasoline, kerosene, acetone, oil, industrial alcohol) corresponding to various values of the speed of droplet flow $U_{\mathrm{m}}$ and speed of the opposite flow of high-temperature combustion products $U_{\mathrm{g}}$.

Fig. 2 shows the obtained dependencies of characteristic times $t_{\mathrm{e}}$ on speeds $U_{\mathrm{g}}$ of counter combustion products of studied oil products (for two groups of droplets various in sizes an two values of $\left.U_{\mathrm{m}}\right)$.

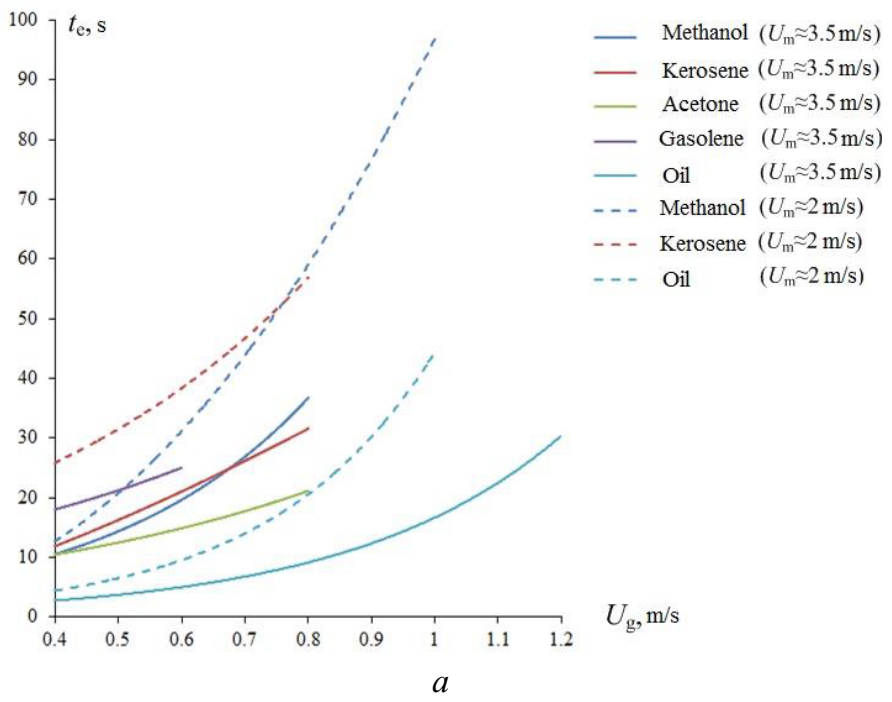




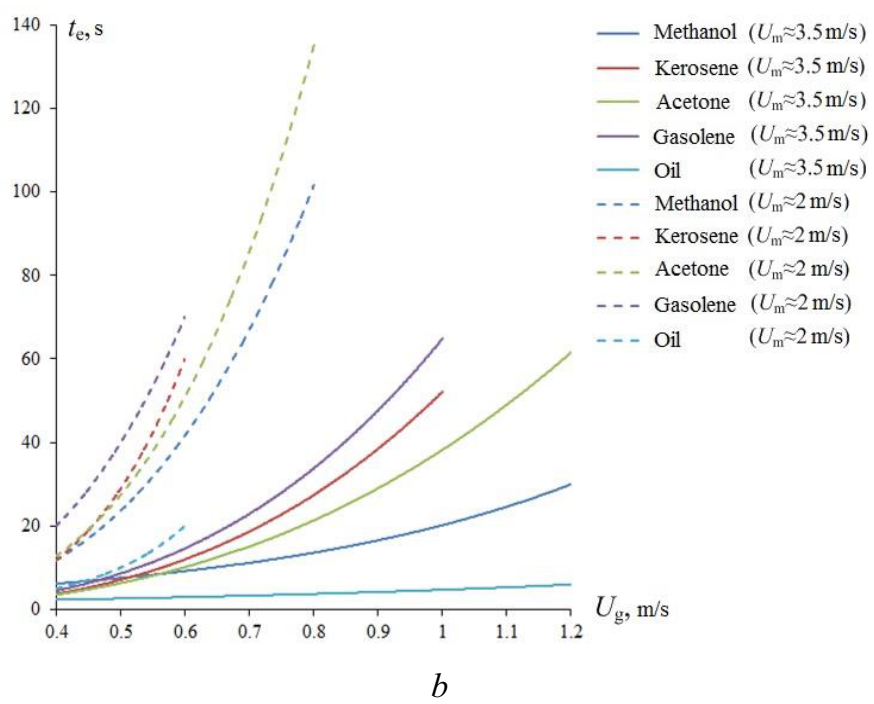

Figure 2. Approximation dependences of characteristic extinguishing times $t_{\mathrm{e}}$ of flammable liquids on the speeds of combustion products $U_{\mathrm{g}}$ for various initial motion speeds of droplets $U_{\mathrm{m}}: a-R_{\mathrm{m}}=0.03-0.12 \mathrm{~mm} ; b-R_{\mathrm{m}}=0.05-$ $0.20 \mathrm{~mm}$ (deficiency or interruption of the curve in the figure corresponds to the conditions under which extinguishing is not reached).

The curves presented in Fig. 2 allow drawing the conclusion of failure in reaching the conditions of the extinguishing of combustible liquids used in experiments by a flow of atomized water if the speed of extinguishing composition droplets is lower than $U_{\mathrm{m}}=3 \mathrm{~m} / \mathrm{s}$. The resulting effect is due, possibly, to the phenomena of braking, turning and entrainment of droplets of atomized extinguishing liquid by the high-temperature combustion products moving opposite (features of the atomized liquid droplets moving in the hot gases were studied in the references $[3,10])$. Perhaps, the relatively low initial speed of extinguishing composition droplets does not allow them to reach the area of the flame. The stock of the kinetic energy of droplets at an initial rate $U_{\mathrm{m}}=3 \mathrm{~m} / \mathrm{s}$ makes it possible for the atomized flow to reach the area of the combustion source, reduce the temperature in this area and expel the oxidizer from the flame zone formed as a result of the phase transition of vapor cloud.

Also, according to the results of the researches for the two groups of characteristic droplet size, we obtained the dependences of the composition of the extinguishing composition on the speed of hightemperature gas flow $U_{\mathrm{g}}$ for different values of the initial velocity of water droplets $U_{\mathrm{m}}$ (Fig. 3).

The analysis of dependences presented in Fig. 3 allows concluding that the most favorable prognosis in extinguishing by atomized water flow among that flammable liquids used in the experiments include is typical of oil. When extinguishing the fires by droplet flows of both dispersion groups, it was possible to stifle a fire with less consumption of extinguishing medium (Fig. 3). Probably, the result is connected to a high temperature of evaporation of oil. As is well known [11], the oxidation (burning) of liquids occurs in the gas phase. The oil evaporates intensively at considerably higher temperatures (about $500 \mathrm{~K}$ ) than the corresponding temperatures of other flammable liquids. As a result of the thermo-physical properties, to maintain the concentration of oil vapors in the level required for a stable combustion, the temperatures in the source must comply with the temperature of intensive evaporation. When extinguishing this fire by a flow of atomized water, the injection of extinguishing composition in the flame zone reduces quickly the temperature of the source up to values which are lower than the temperature of intensive evaporation of oil. 

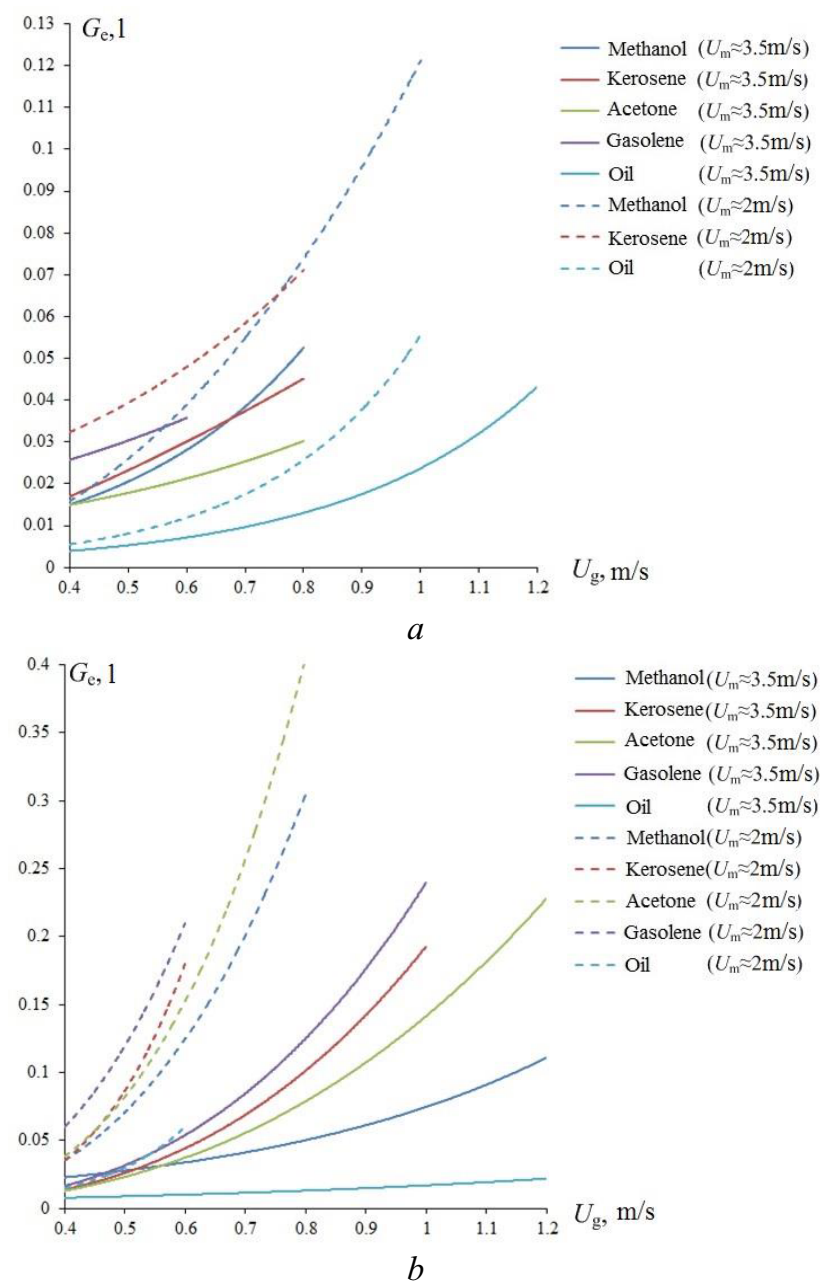

Figure 3. Approximation dependences of characteristic consumptions $V_{\mathrm{e}}$ for extinguishing the flammable liquids on the speeds of combustion products $U_{\mathrm{g}}$ for various initial speeds of droplet movement $U_{\mathrm{m}}: a-R_{\mathrm{m}}=0.03-0.12$ $\mathrm{mm} ; b-R_{\mathrm{m}}=0.05-0.20 \mathrm{~mm}$ (deficiency or interruption of the curve in the figure corresponds to the conditions under which extinguishing is not reached).

\section{Conclusions}

The conducted experimentation investigations allowed defining the typical times for extinguishing different flammable liquids by a flow of atomized water of various dispersability. Also, we determined the extreme values of initial efflux speed for droplets of atomized extinguishing composition at which the conditions of flame suppression of combustible liquids are provided. The results of conducted researches allow us to conclude about possibility in fire suppression of liquid fuels and organic flammable liquids by flows of atomized water, and to formulate the recommendations on the selection of dispersion of droplets, the duration of exposure to the heat source, and estimate the costs of extinguishing medium. The obtained data for features of extinguishing the fires of oil and oil products by atomized water flow can be used for development and improvement of the polydisperse fire extinguishing systems. 


\section{Acknowledgments}

This work was supported by the Russian Foundation for Basic Research (project 15-38-20006).

\section{References}

1. S.V. Sobur, Pozharnaya bezopasnost' neftegazokhimicheskikh predpriyatiy: Spravochnik [Fire safety in petrochemical enterprises: Guide]. Moskow, PozhKniga Publ., 2004 (in Russian).

2. V.I. Gorshkov, Tusheniye plameni goryuchikh zhidkostey [Extinguishing the flame of combustible liquids]. Moskow, Pozhnauka Publ., 2007 (in Russian).

3. R.S. Volkov, G.V. Kuznetsov, P.A. Strizhak, Int. J. Heat and Mass Trans. 79 (2014).

4. D.O. Glushkov, G.S. Nyashina, P.A. Strizhak, R.S. Volkov, IOP Conf. Series: Earth and Environmental Science. 27 (2015).

5. G.V. Kuznetsov, M.V. Piskunov, P.A. Strizhak, Int. J. Heat and Mass Trans. 92 (2016).

6. R.D. Keane, R.J. Adrian, Appl. Sci. Res. 49 (1992).

7. D. Damiani, E. Meillot, D. Tarlet, J. Therm. Spray Technol. 23, 3 (2014).

8. M. Legrand, J. Nogueira, A. Lecuona, S. Nauri, P.A. Rodríguez, Exp. Fluids 48, 5 (2010).

9. R.S. Volkov, G.V. Kuznetsov, P.A. Strizhak, Int. J. Heat Mass Transfer 85 (2015).

10. O.V. Vysokomornaya, G.V. Kuznetsov, P.A. Strizhak, Fire Saf. Journ. 70 (2014).

11. V.N. Vilyunov, V.E. Zarko, Ignition of Solids, Elsevier, Amsterdam, 1989. 